\title{
Twisted surfaces with null rotation axis in Minkowski 3-space
}

\author{
Wendy Goemans · Ignace Van de \\ Woestyne
}

For online version, see http://link.springer.com/article/10.1007/s00025-015-0462-2

\begin{abstract}
We examine curvature properties of twisted surfaces with null rotation axis in Minkowski 3-space. That is, we study surfaces that arise when a planar curve is subject to two synchronized rotations, possibly at different speeds, one in its supporting plane and one of this supporting plane about an axis in the plane. Moreover, at least one of the two rotation axes is a null axis. As is clear from its construction, a twisted surface generalizes the concept of a surface of revolution.

We classify flat, constant Gaussian curvature, minimal and constant mean curvature twisted surfaces with a null rotation axis. Aside from pseudospheres, pseudohyperbolic spaces and cones, we encounter B-scrolls in these classifications. The appearance of B-scrolls in these classifications is of course the result of the rotation about a null axis. As for the cones in the classification of flat twisted surfaces, introducing proper coordinates, we prove that they are determined by so-called Clelia curves. With a Clelia curve we mean a curve that has linear dependent spherical coordinates.
\end{abstract}

Keywords twisted surface $\cdot$ null rotation axis $\cdot$ constant Gaussian curvature minimal surface $\cdot$ constant mean curvature $\cdot$ B-scroll

Mathematics Subject Classification (2000) MSC 53A35 - MSC 53A10

W. Goemans

KU Leuven, Warmoesberg 26, 1000 Brussel, Belgium

Tel.: +32-2-6098813

Fax: $+32-2-2176464$

E-mail: wendy.goemans@kuleuven.be

I. Van de Woestyne

KU Leuven, Warmoesberg 26, 1000 Brussel, Belgium

E-mail: ignace.vandewoestyne@kuleuven.be 


\section{Introduction}

A twisted surface arises when a planar curve is rotated in its supporting plane while simultaneously this supporting plane rotates about a containing axis, possibly at a different rotation speed. These twisted surfaces were introduced in [7] to generalize two special surfaces, namely, the Möbius strip and the twisted Klein bottle. Although a twisted surface is a natural generalization of a surface of revolution, it seems, to the best of our knowledge, that twisted surfaces are hardly studied. Only in [12], the orientability and power series of twisted surfaces were studied.

In $[5,6]$, we already partially filled this gap by studying curvature properties of twisted surfaces in Euclidean 3-space and of regular twisted surfaces, that is, twisted surfaces with non-null rotation axes in Minkowski 3-space. In those articles, we obtained classifications of flat, constant Gaussian curvature and constant mean curvature (regular) twisted surfaces. We also showed that there exists no minimal (regular) twisted surface in Euclidean or in Minkowski 3space when excluding the surfaces of revolution.

Since constructions using null subspaces often lead to deviating results, we treat these separately in this article. Indeed, when studying here twisted surfaces in Minkowski 3-space with null rotation axis having constant Gaussian curvature or constant mean curvature, we encounter new examples. One of these examples is a B-scroll, which is a flat and minimal twisted surface.

The multi-rotation surfaces studied in [4] can be considered as being more general analogues of the twisted surfaces treated here. These multi-rotation surfaces generalize rotation hypersurfaces by allowing higher dimensional profiles which are rotated consecutively about higher dimensional 'axes' lying in orthogonal subspaces.

Also note the similarity of the construction of a twisted surface and that of a generalized helicoid or a helicoidal surface. Indeed, a generalized helicoid is obtained by rotating a planar curve about an axis in its supporting plane while simultaneously translating this curve in the direction of the rotation axis, see e.g. [7].

The remainder of this text is as follows. First, some preliminaries are stated. Then, the construction of a singular twisted surface in Minkowski 3-space is explained whereafter curvature properties for these surfaces are studied.

\section{Preliminaries}

When we equip $\mathbb{R}^{3}$ with the indefinite Minkowski metric $\langle\mathbf{v}, \mathbf{w}\rangle=v_{1} w_{1}+$ $v_{2} w_{2}-v_{3} w_{3}$ for $\mathbf{v}=\left(v_{1}, v_{2}, v_{3}\right)$ and $\mathbf{w}=\left(w_{1}, w_{2}, w_{3}\right)$ in $\mathbb{R}^{3}$, the resulting Minkowski space is denoted by $\mathbb{E}_{1}^{3}$.

In $\mathbb{E}_{1}^{3}$, a vector has one of three different causal characters. Namely, a vector $\mathbf{v}$ in $\mathbb{E}_{1}^{3}$ is spacelike if $\langle\mathbf{v}, \mathbf{v}\rangle>0$, it is timelike if $\langle\mathbf{v}, \mathbf{v}\rangle<0$ and it is null or lightlike if $\langle\mathbf{v}, \mathbf{v}\rangle=0$ and $\mathbf{v} \neq 0$. As a consequence, also a plane in $\mathbb{E}_{1}^{3}$ has one of these different causal characters: 
- it is spacelike if it contains no timelike and no null vector, or equivalently, if its normal is timelike,

- it is timelike if it contains a timelike vector, or equivalently, if it contains two linearly independent null vectors, or equivalently, if its normal is spacelike,

- it is null if it contains a null vector and no timelike vector, or equivalently, if its normal is null.

See, e.g. [11] for these equivalences. Because of this choice of metric, the $x$-axis and the $y$-axis are spacelike, while the $z$-axis is timelike. Hence, the $y z$-plane and $x z$-plane are timelike, while the $x y$-plane is spacelike.

In order to construct a twisted surface in $\mathbb{E}_{1}^{3}$, we need to know how a rotation about an axis in $\mathbb{E}_{1}^{3}$ is described. From [3] we list here the rotations useful for determining twisted surfaces with null rotation axis. A rotation about the spacelike $y$-axis and one about the null axis $\left\{\begin{array}{l}x=z \\ y=0\end{array}\right.$ is given by

$$
\left(\begin{array}{ccc}
\cosh s & 0 & \sinh s \\
0 & 1 & 0 \\
\sinh s & 0 & \cosh s
\end{array}\right) \text { and }\left(\begin{array}{ccc}
1-\frac{s^{2}}{2} & s & \frac{s^{2}}{2} \\
-s & 1 & s \\
-\frac{s^{2}}{2} & s & 1+\frac{s^{2}}{2}
\end{array}\right) \text {, respectively. }
$$

\section{Twisted surfaces with null rotation axis in $\mathbb{E}_{1}^{3}$}

To construct a twisted surface, we start with a planar curve $\alpha$ called the profile curve, and perform two synchronized rotations on it, possibly at different speeds. The profile curve rotates in its supporting plane about an axis that is perpendicular to the plane. Simultaneously, the supporting plane is rotated about an axis that lies in the plane.

Up to a transformation, this construction leaves only one possible parameterization of a twisted surface in $\mathbb{E}^{3}$, see [5] or [6]. In $\mathbb{E}_{1}^{3}$, several different possibilities must be considered because the supporting plane of the profile curve can have different causal characters as well as the rotation axes. We have the following overview:

1. The supporting plane of the profile curve is spacelike. In this case the rotation axis perpendicular to the supporting plane is timelike and the rotation axis in the supporting plane is spacelike. Hence, this leads to a first possible construction of a twisted surface in $\mathbb{E}_{1}^{3}$.

2. The supporting plane of the profile curve is timelike. Then, the rotation axis perpendicular to the supporting plane is spacelike and the rotation axis in the supporting plane can have either causal character:
(a) spacelike,
(b) timelike,
(c) null,
hence leading to three other possible constructions of twisted surfaces in $\mathbb{E}_{1}^{3}$. 
3. The supporting plane of the profile curve is null. Thus, the rotation axis perpendicular to the supporting plane is also null and the rotation axis in the supporting plane can be

(a) null,

(b) spacelike,

leading to two last possible constructions of twisted surfaces in $\mathbb{E}_{1}^{3}$.

Because of these different possibilities, we introduced the following definition in [5].

Definition 1 A twisted surface in $\mathbb{E}_{1}^{3}$ is regular if both the rotation axes are non-null. Otherwise we call the twisted surface singular.

The regular twisted surfaces in $\mathbb{E}_{1}^{3}$ arise from cases $1,2 \mathrm{a}$ and $2 \mathrm{~b}$ and these are already studied in [5] and [6]. Both publications provide examples of regular twisted surfaces in $\mathbb{E}_{1}^{3}$. Here, we examine singular twisted surfaces which result from cases 2c, 3a and 3b. As we will show in the proof of Theorem 2, the pseudospheres and the pseudohyperbolic spaces in $\mathbb{E}_{1}^{3}$ can be parameterized as (singular) twisted surfaces.

3.1 The profile curve lies in a timelike plane

Without losing generality, we can assume that the profile curve $\alpha$ lies in the $x z$-plane and is parameterized as $\alpha(t)=(f(t), 0, g(t))$. Rotate $\alpha$ about the axis through $(a, 0,0)$ parallel with the $y$-axis:

$$
\begin{aligned}
\left(\begin{array}{l}
a \\
0 \\
0
\end{array}\right)+\left(\begin{array}{ccc}
\cosh (b s) & 0 & \sinh (b s) \\
0 & 1 & 0 \\
\sinh (b s) & 0 \cosh (b s)
\end{array}\right) & \left(\begin{array}{c}
f(t) \\
0 \\
g(t)
\end{array}\right) \\
= & \left(\begin{array}{c}
a+f(t) \cosh (b s)+g(t) \sinh (b s) \\
0 \\
f(t) \sinh (b s)+g(t) \cosh (b s)
\end{array}\right) .
\end{aligned}
$$

Simultaneously, rotate about the null axis $\left\{\begin{array}{l}x=z \\ y=0\end{array}\right.$. That is,

$$
\mathbf{x}(s, t)=\left(\begin{array}{ccc}
1-\frac{s^{2}}{2} & s & \frac{s^{2}}{2} \\
-s & 1 & s \\
-\frac{s^{2}}{2} & s & 1+\frac{s^{2}}{2}
\end{array}\right)\left(\begin{array}{c}
a+f(t) \cosh (b s)+g(t) \sinh (b s) \\
0 \\
f(t) \sinh (b s)+g(t) \cosh (b s)
\end{array}\right)
$$

parametrizes a singular twisted surface in $\mathbb{E}_{1}^{3}$. Here $a, b \in \mathbb{R}$ and the parameter $b$ allows the two rotations to have different rotation speeds. This construction is sketched in Figure 1. If $b=0$, the twisted surfaces reduces to a surface of revolution with a null rotation axis which we call here a standard parameterized surface of revolution. For a study of these standard parameterized surfaces of revolution, see e.g. [2] and [8]. Using numerical methods, the authors in [9] and [10] calculated explicit examples of constant mean curvature surfaces of revolution with a null rotation axis in $\mathbb{E}_{1}^{3}$. 


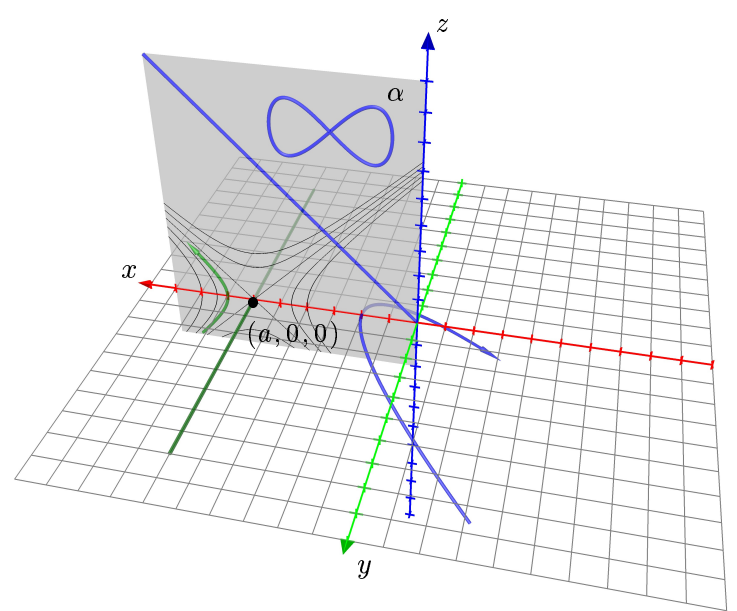

Fig. 1 Initial setting resulting in the parameterization of a singular twisted surface.

3.2 The profile curve lies in a lightlike plane

3.2.1 Two rotations about the same null axis

Assume, without losing generality, that the profile curve $\alpha$ lies in the null plane $x=z$ and is parameterized as $\alpha(t)=(f(t), g(t), f(t))$. The null axis perpendicular to this null plane is the axis $\left\{\begin{array}{l}x=z \\ y=0\end{array}\right.$. This axis also lies in the plane, and no other null axis linearly independent from this axis lies in the null plane (otherwise the plane would be timelike). Thus, rotating about two null axes amounts to rotating twice about the same null axis. But, then this twisted surface is an ordinary surface of revolution. Moreover, it is degenerate.

\subsubsection{Rotate about a null axis and about a spacelike axis}

In this case, use again the null axis $\left\{\begin{array}{l}x=z \\ y=0\end{array}\right.$, the point $(a, c, a)$ and the spacelike $y$-axis, leading to the parameterization

$$
\mathbf{x}(s, t)=(a+f(t)+b s g(t))(\cosh s+\sinh s)(1,0,1)+(g(t)+c)(0,1,0),
$$

with $a, b, c \in \mathbb{R}$. But, also this surface is degenerate.

Therefore, we have the following definition.

Definition $2 \mathrm{Up}$ to a transformation, a singular twisted surface in $\mathbb{E}_{1}^{3}$ is a surface parameterized by (1). 


\section{Constant curvature singular twisted surfaces}

We calculate and examine the Gaussian curvature and the mean curvature of a singular twisted surface. For details involving patch computations, see e.g. [11]. The Gaussian curvature is

$$
K(s, t)=\langle\mathbf{U}(s, t), \mathbf{U}(s, t)\rangle \frac{L(s, t) N(s, t)-M(s, t)^{2}}{E(s, t) G(s, t)-F(s, t)^{2}}
$$

and the mean curvature is

$$
H(s, t)=\frac{E(s, t) N(s, t)-2 F(s, t) M(s, t)+G(s, t) L(s, t)}{2\left(E(s, t) G(s, t)-F(s, t)^{2}\right)}
$$

where $U(s, t)$ is the unit normal to the surface, $E(s, t), F(s, t)$ and $G(s, t)$ are the components of the first fundamental form and $L(s, t), M(s, t)$ and $N(s, t)$ are the components of the second fundamental form. To save space, we do not explicitly write down the Gaussian curvature and the mean curvature of a singular twisted surface, since these are huge expressions.

To make the calculations less elaborate, we assume from here on, without losing generality, that the profile curve $\alpha$ is parameterized as $\alpha(t)=(t, 0, g(t))$. Then, a singular twisted surface is degenerate if and only if $g(t)= \pm t$, which we exclude from here on. Also, we assume that $b \neq 0$ in parameterization (1) since otherwise the singular twisted surface reduces to a standard parameterized surface of revolution, which was already studied in [2] (constant Gaussian curvature) and in [8] (constant mean curvature surfaces, including minimal surfaces of revolution).

Assuming that a singular twisted surface has constant Gaussian curvature or constant mean curvature leads to an equation of the form

$$
\sum_{i=0}^{n+1} A_{i}(t) \cosh ^{i}(b s)+\sum_{i=0}^{n} B_{i}(t) \cosh ^{i}(b s) \sinh (b s)=0
$$

with $A_{i}(t)$ and $B_{i}(t)$ expressions involving the unknown function $g$. Because the hyperbolic cosine and hyperbolic sine functions are linearly independent, one obtains the following differential equations in $g$,

$$
A_{i}(t)=0 \text { for } i \in\{0,1, \ldots, n, n+1\} \text { and } B_{j}(t)=0 \text { for } j \in\{0,1, \ldots, n\} \text {. }
$$

We use the computer algebra system Maple to compute the Gaussian curvature and the mean curvature of a singular twisted surface as well as for calculating and manipulating the equations (2). To condense the notation slightly, we do not always explicitly write the parameter $t$ of the function $g$ in the remainder of this article. 
4.1 Flat singular twisted surfaces

A flat surface is a surface that has vanishing Gaussian curvature everywhere. We classify flat singular twisted surfaces.

Theorem 1 If the standard parameterized surfaces of revolution are excluded, then a singular twisted surface in $\mathbb{E}_{1}^{3}$ is flat if and only if it is (a part of) either

1. a ruled surface parameterized by

$$
\begin{array}{r}
\mathbf{x}(s, t)=\left(a\left(1-\frac{s^{2}}{2}\right)+\frac{c s^{2}}{2} e^{-b s}+c \sinh (b s)+t e^{b s},-a s+c s e^{-b s}\right. \\
\left.-a \frac{s^{2}}{2}+\frac{c s^{2}}{2} e^{-b s}+c \cosh (b s)+t e^{b s}\right)
\end{array}
$$

where $a, b, c \in \mathbb{R}$ and $b c \neq 0$.

2. a cone over the curve

$$
\begin{aligned}
\left(\cosh (b s)+c \sinh (b s)+(c-1) \frac{s^{2}}{2} e^{-b s}, s(c-1) e^{-b s}\right. & \\
& \left.\sinh (b s)+c \cosh (b s)+(c-1) \frac{s^{2}}{2} e^{-b s}\right)
\end{aligned}
$$

with vertex the origin. Here $b, c \in \mathbb{R}$ and $b \neq 0$ and $c \neq \pm 1$.

Proof Expressing that $K(s, t)=0$ for a singular twisted surface, results in $n=4$ in Equations (2) and

$$
A_{4}(t)=0 \quad \Leftrightarrow \quad g^{\prime \prime}(g-t)^{3}\left(g^{\prime}-1\right)=0 .
$$

Thus, we can assume that $g(t)=c t+d$ and use this in the other Equations (2). Then, we obtain that

$$
A_{2}(t)=0 \quad \Leftrightarrow \quad b^{2} d^{2}(c-1)^{2}=0 .
$$

If $c=1$ then $d$ cannot be zero since otherwise the surface is degenerate. Therefore, we obtain the first parameterization in the statement of the theorem, a ruled surface.

If $d=0$, then, $c \neq \pm 1$ since otherwise the surface is degenerate. But, since then

$$
A_{0}(t)=0 \quad \Leftrightarrow \quad a^{2} b^{2}(c-1)^{2}(c+1)^{2}=0,
$$

we have $a=0$ and we find the cone as stated in the theorem.

Remark 1 The ruled surface in Theorem 1 has a lightlike ruler. We summarize here how this kind of ruled surfaces is treated in [1]. Namely, it can be reparameterized in such a way that it suits the definition of a B-scroll. That is, a ruled surface parameterized by $x(s, t)=\gamma(s)+t B(s)$ is a null scroll if $\left\langle\gamma^{\prime}(s), \gamma^{\prime}(s)\right\rangle=0=\langle B(s), B(s)\rangle$ and $\left\langle\gamma^{\prime}(s), B(s)\right\rangle=-1$. If $\gamma^{\prime}$ is non-null and $B$ is null, then after a reparameterization, the surface is a null scroll. 


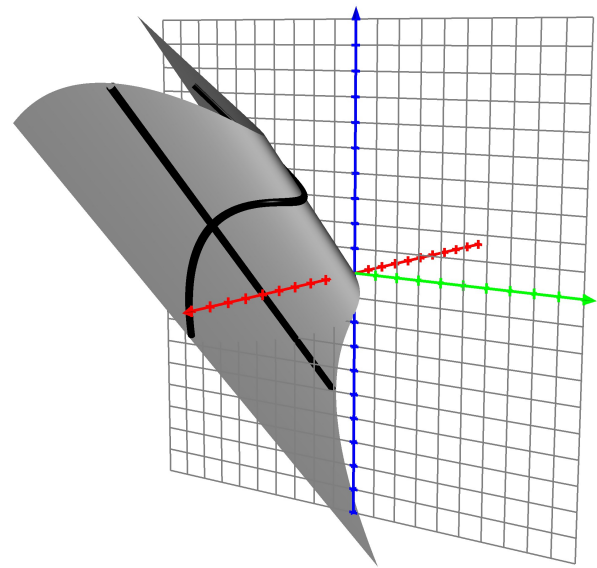

(a) A B-scroll as parameterized in Theorem 1 with $a=\frac{3}{2}, b=\frac{1}{2}$ and $c=1$.

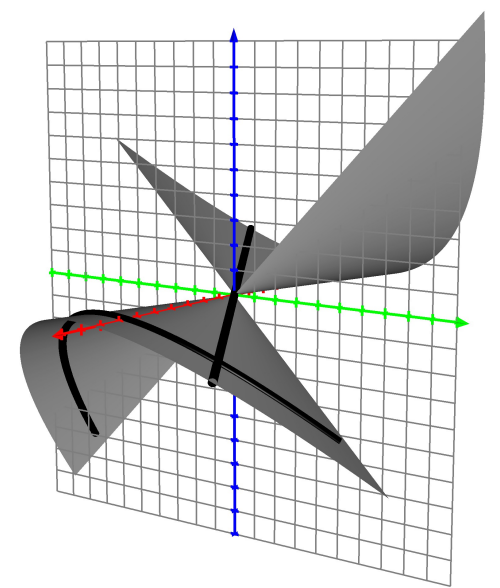

(b) A cone over a Clelia curve as parameterized in Theorem 1 with $b=\frac{1}{8}$ and $c=0$.

Fig. 2 Flat singular twisted surfaces.

Moreover, for a null scroll, using a particular frame field, one has $B^{\prime}(s) \times$ $B(s)=-w(s) B(s)$. If $w(s)$ is constant, then the null scroll is called a $B$-scroll.

Also for a null scroll, $K(s, t)=w(s)^{2}$ and $H(s, t)=w(s)$. This is also shown in [3] and as is concluded there, every null scroll is a Weingarten surface. That is, there exists a functional relation between the Gaussian curvature and the mean curvature of the surface, which is easily seen to be $K=H^{2}$ for a null scroll.

Thus, in Theorem 1, we find a B-scroll which is flat and minimal. See Figure 2(a) for a drawing of such a B-scroll.

Remark 2 The curve determining the cone in Theorem 1, parameterized by (3), lies on a pseudosphere of radius $\sqrt{1-c^{2}}$ if $|c|<1$ and on a pseudohyperbolic space of radius $\sqrt{c^{2}-1}$ if $|c|>1$. This is similar to the results for flat regular twisted surfaces in [5]. Using the proper "spherical" coordinates, it can be shown that this curve is also a Clelia curve, that is, a curve which has linear dependent "spherical" coordinates. We show this in the case the curve parameterized by (3) lies on a pseudosphere. The pseudosphere can be parameterized using the following "spherical" coordinates, adapted to the null case,

$$
\begin{aligned}
\left(\cosh u\left(1-\frac{v^{2}}{2}\right)+\sinh u \frac{v^{2}}{2},-v \cosh u\right. & +v \sinh u \\
& \left.-\cosh u \frac{v^{2}}{2}+\sinh u\left(1+\frac{v^{2}}{2}\right)\right),
\end{aligned}
$$


obtained from rotating the curve $(\cosh u, 0, \sinh u)$ about the null axis $\left\{\begin{array}{l}x=z \\ y=0\end{array}\right.$. Note that this can be simplified to

$$
\left(\cosh u-\frac{v^{2}}{2} e^{-u},-v e^{-u}, \sinh u-\frac{v^{2}}{2} e^{-u}\right) .
$$

Now, choose $\gamma \in \mathbb{R}$ such that $\sinh \gamma=\frac{c}{\sqrt{1-c^{2}}}$ and $\cosh \gamma=\frac{1}{\sqrt{1-c^{2}}}$. Then, (3) can be rewritten as

$$
\begin{aligned}
\frac{1}{\cosh \gamma}\left(\cosh (b s+\gamma)+\frac{c-1}{\sqrt{1-c^{2}}} \frac{s^{2}}{2} e^{-b s},\right. & \frac{c-1}{\sqrt{1-c^{2}}} s e^{-b s} \\
& \left.\sinh (b s+\gamma)+\frac{c-1}{\sqrt{1-c^{2}}} \frac{s^{2}}{2} e^{-b s}\right) .
\end{aligned}
$$

Noting that

$$
\frac{c-1}{\sqrt{1-c^{2}}}=\sinh \gamma-\cosh \gamma=-e^{-\gamma}
$$

this is rewritten as follows

$$
\frac{1}{\cosh \gamma}\left(\cosh (b s+\gamma)-\frac{s^{2}}{2} e^{-(b s+\gamma)},-s e^{-(b s+\gamma)}, \sinh (b s+\gamma)-\frac{s^{2}}{2} e^{-(b s+\gamma)}\right) \text {. }
$$

Applying a rotation about the null axis $\left\{\begin{array}{l}x=z \\ y=0\end{array}\right.$ with rotation angle $\frac{\gamma}{b}$ transforms this parameterization to

$$
\begin{aligned}
\frac{1}{\cosh \gamma}\left(\cosh (b s+\gamma)-\frac{1}{2}\left(s+\frac{\gamma}{b}\right)^{2} e^{-(b s+\gamma)},-\left(s+\frac{\gamma}{b}\right) e^{-(b s+\gamma)}\right. \\
\left.\sinh (b s+\gamma)-\frac{1}{2}\left(s+\frac{\gamma}{b}\right)^{2} e^{-(b s+\gamma)}\right)
\end{aligned}
$$

from which the linear dependency of the spherical coordinates is easily observed.

See Figure 2(b) for a drawing of a cone over such a Clelia curve.

4.2 Non-flat constant Gaussian curvature singular twisted surfaces

We classify singular twisted surfaces that have non-zero constant Gaussian curvature.

Theorem 2 If the standard parameterized surfaces of revolution are excluded, then a singular twisted surface in $\mathbb{E}_{1}^{3}$ has non-zero constant Gaussian curvature $K$ if and only if it is either a (part of a) pseudosphere (when $K>0$ ) or $a$ pseudohyperbolic space (when $K<0$ ), in each case of radius $\frac{1}{\sqrt{|K|}}$. 
Proof The condition $K(s, t)=k$ with $k \in \mathbb{R}_{0}$ leads to $n=4$ in Equations (2) and

$$
\begin{aligned}
& A_{4}(t)=0 \Leftrightarrow(g-t)^{3}\left(g^{\prime}-1\right)\left(g^{\prime \prime}-k\left(g^{\prime}-1\right)\left(g^{\prime}+1\right)^{2}(g-t)\right)=0 . \\
& \text { If } g(t)=t+c \text { with } c \in \mathbb{R}_{0} \text {, a contradiction follows from }
\end{aligned}
$$

$$
A_{0}(t)=0 \quad \Leftrightarrow \quad b^{4} c^{4} k=0 .
$$

Hence, the last term in Equation (4) is zero, from which we can solve an expression for $g^{\prime \prime}(t)$. Use that in the other Equations (2), then

$$
A_{3}(t)=0 \quad \Leftrightarrow \quad a k(g-t)^{3}\left(g^{\prime}-1\right)^{2}\left(g^{\prime}+1\right)^{2}=0 .
$$

Since we already examined $g^{\prime}(t)=1$ in the previous case, two subcases must be considered.

Case $1 a=0$.

With this,

$$
\begin{array}{r}
A_{2}(t)=0 \Leftrightarrow \quad b^{2}\left(g^{\prime}-1\right)^{2}\left(t g^{\prime}-g\right)\left(\left(k g^{3}-k t g^{2}-k t^{2} g+k t^{3}-t\right) g^{\prime}\right. \\
\left.+k g^{3}-k t g^{2}-k t^{2} g+k t^{3}+g\right)=0 .
\end{array}
$$

Again, two subcases must be considered.

Case 1 A $g(t)=c t$ with $c \in \mathbb{R}$ and $c \neq \pm 1$.

But, from

$$
A_{0}(t)=0 \quad \Leftrightarrow \quad b^{4} k t^{4}(c-1)^{4}(c+1)^{4}=0
$$

only a contradiction is found.

Case $1 B$ The last term of Equation (5) is zero. Denote this by $C g^{\prime}+D=0$. If $C=0$, then also $D=0$ and a contradiction follows directly from $D-C=$ $g+t=0$.

If $C \neq 0$, we solve $g^{\prime}=-\frac{D}{C}$ and use it in the other Equations (2). Then,

$$
A_{0}(t)=0 \quad \Leftrightarrow \quad b^{4} k(g-t)^{4}(g+t)^{4}\left(k g^{2}-k t^{2}+1\right)^{4}=0 .
$$

So, $g(t)= \pm \sqrt{t^{2}-\frac{1}{k}}$. Hence, the twisted surface is a pseudosphere or a pseudohyperbolic space. Indeed, assume for instance that $k>0$. Then, the profile curve $\alpha(t)=\left(t, 0, \pm \sqrt{t^{2}-\frac{1}{k}}\right)$ is rewritten using $\sqrt{k} t=\cosh \bar{t}$ and $\pm \sqrt{k} \sqrt{t^{2}-\frac{1}{k}}=\sinh \bar{t}$ as $\alpha(\bar{t})=\frac{1}{\sqrt{k}}(\cosh \bar{t}, 0, \sinh \bar{t})$. Rotating this curve about the $y$-axis leads to $\frac{1}{\sqrt{k}}(\cosh (\bar{t}+b s), 0, \sinh (\bar{t}+b s))$. Denoting with a new parameter $T=\bar{t}+b s$ and performing a rotation about the null axis $\left\{\begin{array}{l}x=z \\ y=0\end{array}\right.$ results in the parameterization of a pseudosphere as mentioned in Remark 2.

Case $2 g(t)=-t+c$ with $c \in \mathbb{R}_{0}$.

In this case

$$
A_{2}(t)=0 \quad \Leftrightarrow \quad b^{2} c^{2}=0,
$$

yielding a contradiction.

Remark 3 This result is the same as for regular twisted surfaces with non-zero constant Gaussian curvature (see [5]). 
4.3 Minimal singular twisted surfaces

If the mean curvature of a surface vanishes identically, then we call the surface minimal. Also for surfaces in $\mathbb{E}_{1}^{3}$ we keep this name, although in general, a minimal surface in $\mathbb{E}_{1}^{3}$ does not minimize area. Contrary to the non-existence of minimal regular twisted surfaces (see [5]), there do exist minimal singular twisted surfaces in $\mathbb{E}_{1}^{3}$.

Theorem 3 If the standard parameterized surfaces of revolution are excluded, then a singular twisted surface in $\mathbb{E}_{1}^{3}$ is minimal if and only if it is (a part of) the B-scroll in Theorem 1.

Proof For $H(s, t)=0$, in Equations (2) $n=3$ and

$$
A_{3}(t)=0 \quad \Leftrightarrow \quad(g-t)^{2}\left((g-t) g^{\prime \prime}+\left(g^{\prime}+1\right)\left(g^{\prime}-1\right)^{2}\right)=0 .
$$

From this, we solve an expression for $g^{\prime \prime}$ which we use in the other equations, then

$$
A_{2}(t)=0 \quad \Leftrightarrow \quad a\left(g^{\prime}+1\right)\left(g^{\prime}-1\right)^{2}(g-t)=0 .
$$

Thus, we have to examine three cases.

Case $1 g(t)=-t+c$ with $c \in \mathbb{R}_{0}$

Then, $B_{0}(t)=0 \Leftrightarrow b^{2} c^{2}=0$ hence, we obtain a contradiction.

Case $2 g(t)=t+c$ with $c \in \mathbb{R}_{0}$.

That is, we find the B-scroll in Theorem 1.

$$
\text { Case } 3 a=0
$$

Now,

$$
\begin{aligned}
& A_{1}(t)=0 \Leftrightarrow \\
& \quad b^{2}\left(g^{\prime}-1\right)\left(\left(g^{2}-t g+2 t^{2}\right) g^{2}+\left(g^{2}-6 t g+t^{2}\right) g^{\prime}+2 g^{2}-t g+t^{2}\right)=0 .
\end{aligned}
$$

Since we already examined the case $g^{\prime}(t)=1$, the last term of $A_{1}(t)$ must be zero. But, if it is considered as a polynomial in $g^{\prime}(t)$, then, it turns out the discriminant is negative, hence, there is no real solution for $g^{\prime}(t)$.

\subsection{Constant mean curvature singular twisted surfaces}

A surface that has constant mean curvature is called a CMC surface. The classification of CMC singular twisted surfaces in $\mathbb{E}_{1}^{3}$ turns out to be analogous to that of regular twisted surfaces in $\mathbb{E}_{1}^{3}$ (see [5]).

Theorem 4 If the standard parameterized surfaces of revolution are excluded, then a singular twisted surface in $\mathbb{E}_{1}^{3}$ is a CMC surface with constant mean curvature $H$ if and only if it is either (a part of) a pseudosphere or of a pseudohyperbolic space, in each case of radius $\frac{1}{|H|}$. 
Proof To classify the CMC twisted surfaces, we work with the square of the equation $H(s, t)=h$ with $h \in \mathbb{R}_{0}$, which leads to $A_{i}(t)=0$ and $B_{j}(t)=0$ with $i \in\{0,1, \ldots, 6\}$ and $j \in\{0,1, \ldots, 5\}$ in Equations (2). Moreover, to be able to solve the differential equations, we also take the derivative of the equation $H(s, t)=h$ with respect to $s$, leading to $C_{i}(t)=0$ and $D_{j}(t)=0$ with $i \in\{0,1,2,3,4\}$ and $j \in\{0,1,2,3\}$ in Equations (2).

Then,

$$
C_{4}(t)=0 \quad \Leftrightarrow \quad a b(g-t)^{3}\left(g^{\prime}+1\right)^{2}\left(g^{\prime}-1\right)^{3}=0 .
$$

The cases in which $g(t)= \pm t+c$ with $c \in \mathbb{R}_{0}$ both directly result in a contradiction.

Hence, $a=0$. Then,

$$
C_{3}(t)=0 \quad \Leftrightarrow \quad b^{3}(g-t)^{2}\left(\ldots g^{\prime \prime}+\ldots\right)=0
$$

where the last term can be considered as a first degree polynomial in $g^{\prime \prime}$. This last term must be zero. If the coefficient of $g^{\prime \prime}$ in that term is zero, then there is no real solution for $g$. If the coefficient of $g^{\prime \prime}$ is non-zero, then we solve an expression for $g^{\prime \prime}$ and use that in the other equations. This leads to

$$
C_{1}(t)=0 \quad \Leftrightarrow \quad b^{5}\left(g^{\prime}-1\right)\left(g g^{\prime}-t\right)^{4}\left(\ldots g^{2}+\ldots g^{\prime}+\ldots\right)=0 .
$$

From this equation, only the solution $g(t)= \pm \sqrt{t^{2}-\frac{1}{h^{2}}}$ is found when also using $A_{0}(t)=0$. Therefore, we find the pseudospheres and the pseudohyperbolic spaces.

\section{Conclusions and further research}

As to be expected, due to the use of a null rotation axis, the classifications of constant curvature singular twisted surfaces contain other surfaces in some cases compared to those of constant curvature regular twisted surfaces in Minkowski 3-space. Namely, combining the present results and those in [5], we found a B-scroll which turns out to be the only minimal twisted surface in Minkowski 3-space when the surfaces of revolution are excluded.

The class of twisted surfaces most likely could lead to other interesting results when studied in other spaceforms for instance. It might also be worthwhile defining and studying twisted hypersurfaces analogously to the notion of hypersurfaces of revolution. Thus, it is clear that twisted surfaces can be the subject of several interesting further research.

Acknowledgements The pictures in this article are made with VisuMath [13]. 


\section{References}

1. Alías L.J., Ferrández A., Lucas P., Meroño M.A.: On the Gauss map of B-scrolls. Tsukuba J. Math. 22, 371-377 (1998)

2. Beneki C., Kaimakamis G., Papantoniou B.J.: A classification of surfaces of revolution with constant Gauss curvature in a 3-dimensional Minkowski space. Bull. Calcutta Math. Soc. 90, 441-458 (1998)

3. Dillen F., Kühnel W.: Ruled Weingarten surfaces in Minkowski 3-space. Manuscripta Math. 98, 307-320 (1999)

4. Dillen F., Nölker S.: Semi-parallelity, multi-rotation surfaces and the helix-property. J. reine angew. Math. 435, 33-63 (1993)

5. Goemans W., Van de Woestyne I.: Constant curvature twisted surfaces in 3-dimensional Euclidean and Minkowski space. Proceedings of the Conference RIGA 2014 Riemannian Geometry and Applications to Engineering and Economics Bucharest, Romania, 117-130 (2014)

6. Goemans W., Van de Woestyne I.: Twisted surfaces in Euclidean and Minkowski 3space. Pure and Applied Differential Geometry: 2013, Van der Veken J., Van de Woestyne I., Verstraelen L. and Vrancken L. (Editors), Shaker Verlag (Aachen, Germany), 143-151 (2013)

7. Gray A., Abbena E. (Editor), Salamon S. (Editor): Modern Differential Geometry of Curves and Surfaces with Mathematica. Chapman \& Hall/CRC, Boca Raton (2006)

8. Ishihara T., Hara F.: Surfaces of revolution in the Lorentzian 3-space. J. Math. Univ. Tokushima 22, 1-13 (1988)

9. Lee S., Varnado J. H.: Spacelike constant mean curvature surfaces of revolution in Minkowski 3-Space. DGDS Differ. Geom. Dyn. Syst. Monogr. 8, 144-165 (2006)

10. Lee S., Varnado J. H.: Timelike surfaces of revolution with constant mean curvature in Minkowski 3-Space. DGDS Differ. Geom. Dyn. Syst. Monogr. 9, 82-102 (2007)

11. O'Neill B.: Semi-Riemannian Geometry. Academic Press, London (1983)

12. Stanilov G., Slavova S.: Classification of some twisted surfaces and power series of such surfaces. C. R. Acad. Bulgare Sci. 59, 6, 593-600 (2006)

13. www.visumath.be 